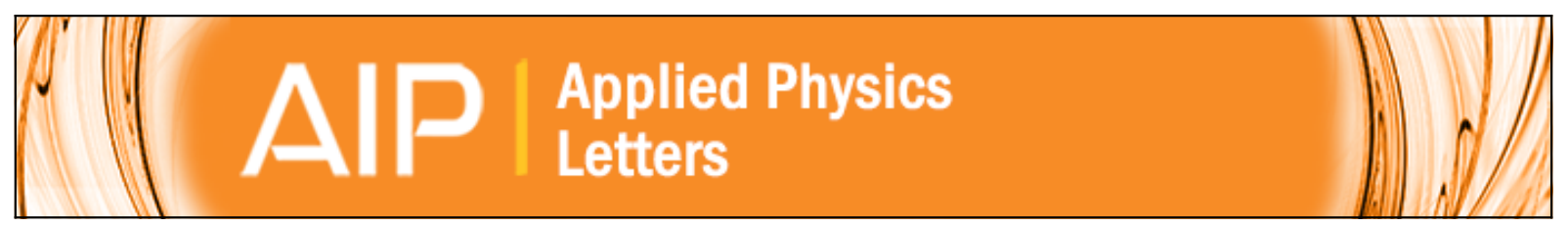

\title{
Asymmetric "melting" and "freezing" kinetics of the magnetostructural phase transition in B2-ordered FeRh epilayers
}

M. A. de Vries, M. Loving, M. McLaren, R. M. D. Brydson, X. Liu, S. Langridge, L. H. Lewis, and C. H. Marrows

Citation: Applied Physics Letters 104, 232407 (2014); doi: 10.1063/1.4883369

View online: http://dx.doi.org/10.1063/1.4883369

View Table of Contents: http://scitation.aip.org/content/aip/journal/apl/104/23?ver=pdfcov

Published by the AIP Publishing

\section{Articles you may be interested in}

Phase ordering and its effect on magnetic and structural properties of FeRh ultrathin films

J. Appl. Phys. 116, 043902 (2014); 10.1063/1.4890032

Magnetic stability of ultrathin FeRh films

J. Appl. Phys. 113, 17C107 (2013); 10.1063/1.4794980

Tuning the magnetostructural phase transition in FeRh nanocomposites

J. Appl. Phys. 113, 023910 (2013); 10.1063/1.4774282

Tailoring the FeRh magnetostructural response with Au diffusion

J. Appl. Phys. 112, 043512 (2012); 10.1063/1.4747921

Surface influenced magnetostructural transition in FeRh films

Appl. Phys. Lett. 95, 222515 (2009); 10.1063/1.3265921

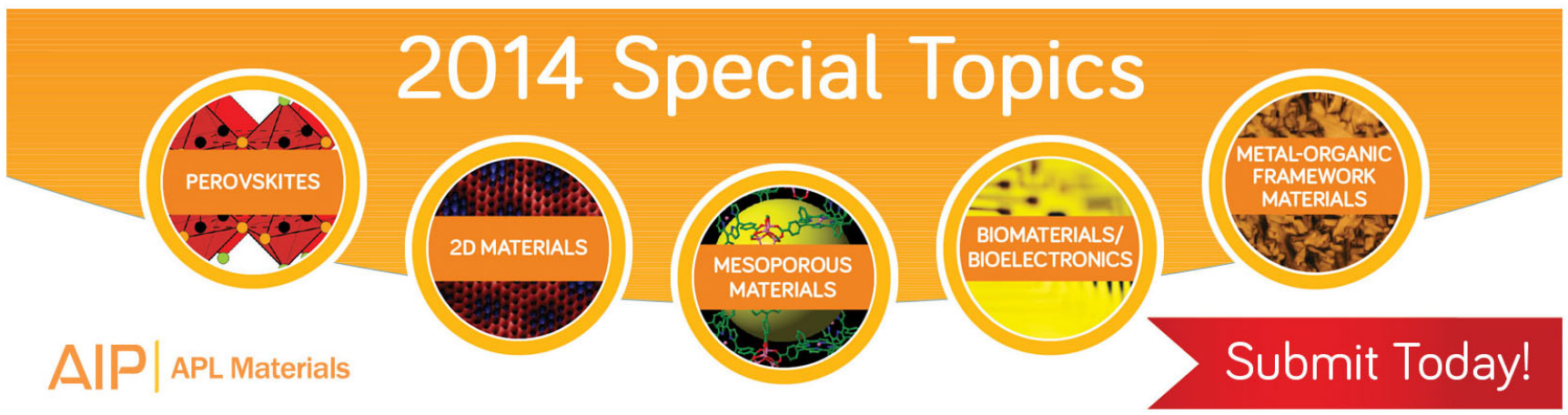




\title{
Asymmetric "melting" and "freezing" kinetics of the magnetostructural phase transition in B2-ordered FeRh epilayers
}

\author{
M. A. de Vries, ${ }^{1,2, a)}$ M. Loving, ${ }^{3}$ M. McLaren, ${ }^{4}$ R. M. D. Brydson, ${ }^{4}$ X. Liu, ${ }^{5,6,7}$ S. Langridge, ${ }^{8}$ \\ L. H. Lewis, ${ }^{3}$ and C. H. Marrows $\left.{ }^{1, b}\right)$ \\ ${ }^{1}$ School of Physics and Astronomy, University of Leeds, Leeds LS2 9JT, United Kingdom \\ ${ }^{2}$ School of Chemistry, University of Edinburgh, Edinburgh EH9 3JJ, United Kingdom \\ ${ }^{3}$ Department of Chemical Engineering, Northeastern University, Boston, Massachusetts 02115, USA \\ ${ }^{4}$ Institute for Materials Research, University of Leeds, Leeds LS2 9JT, United Kingdom \\ ${ }^{5}$ Condensed Matter Physics and Materials Science Department, Brookhaven National Laboratory, Upton, \\ New York 11973, USA \\ ${ }^{6}$ Beijing National Laboratory for Condensed Matter Physics, and Institute of Physics, \\ Chinese Academy of Sciences, Beijing 100190, China \\ ${ }^{7}$ Collaborative Innovation Center of Quantum Matter, Beijing 100871, China \\ ${ }^{8}$ ISIS, Harwell Science and Innovation Campus, Science and Technology Facilities Council, \\ Rutherford Appleton Laboratory, Didcot, Oxon OX11 0QX, United Kingdom
}

(Received 2 May 2014; accepted 28 May 2014; published online 11 June 2014)

\begin{abstract}
Synchrotron X-ray diffraction was used to study the phase transformation processes during the magnetostructural transition in a B2-ordered $\mathrm{FeRh}(001)$-oriented epilayer grown on $\mathrm{MgO}$ by sputtering. Out-of-plane lattice constant measurements within the hysteretic regime of the transition reveal a microstructure consistent with the coexistence of lattice-expanded and contracted phases in spatially distinct regions. It was found that the phase separation is more pronounced during cooling than heating. Furthermore, whilst lattice-expanded domains that span the height of the film can be undercooled by several kelvins, there is no equivalent superheating. This asymmetry between the cooling and heating processes in FeRh is consistent with the difference in the kinetics of generic freezing and melting transitions. ( 2014 AIP Publishing LLC. [http://dx.doi.org/10.1063/1.4883369]
\end{abstract}

Equiatomic B2-ordered FeRh displays an unusual magnetostructural transition from an antiferromagnetic (AF) to a ferromagnetic (FM) state when heated through a critical temperature of $\sim 380 \mathrm{~K}^{1}{ }^{1}$ In bulk samples, the transition is accompanied by a isotropic $1 \%$ volume expansion, ${ }^{2,3}$ a drop in resistivity by typically a factor of two, ${ }^{1}$ and a large entropy release. ${ }^{4}$ The transition also occurs in both epitaxial and polycrystalline FeRh thin films, ${ }^{5-11}$ which are of interest for potential applications in heat-assisted magnetic recording media ${ }^{12}$ or resistive memory cells. ${ }^{13}$ Further research on $\mathrm{FeRh}$ thin films is now focussed on tuning the transition towards specific applications such as by growing epitaxially on a variety of substrates and in heterostructures. ${ }^{8,14-19}$

There are various competing explanations for the underlying cause of these changes to the crystallographic, electronic, and magnetic structure of this material. Ultrafast measurements show that the magnetism can be switched from $\mathrm{AF}$ to $\mathrm{FM}$ within picoseconds with a rapid heat pulse from a laser ${ }^{20}$ much more quickly than the structure responds, ${ }^{21,22}$ ruling out that the change in lattice constant alone causes the change in the magnetic properties. First principles calculations show that there is also an underlying phase transition in the electronic structure, ${ }^{23-27}$ a thesis for which there is growing experimental evidence. ${ }^{11,28-30}$

The magnetostructural phase transition is well-known to be of first order in this material, and as a result shows hysteresis and phase coexistence. The coexistence of the two

\footnotetext{
${ }^{\text {a)} E l e c t r o n i c ~ m a i l: ~ m . a . d e v r i e s @ e d . a c . u k ~}$

b)Electronic mail: c.h.marrows@leeds.ac.uk
}

phases has been inferred from transport measurements, ${ }^{10}$ previous X-ray diffraction experiments, ${ }^{22,31,32} \mathrm{X}$-ray magnetic circular dichroism spectroscopy, ${ }^{33}$ and directly imaged using photoemission electron microscopy (PEEM). ${ }^{17,19,34-36}$ Here, we show that there is an asymmetry in the nature of the phase transition in FeRh between warming and cooling, determined by high-resolution synchrotron X-ray diffraction that revealed the changes in out-of-plane lattice constant of our epilayers. There is greater separation of the coexisting phases (i.e., distinct spatial regions with a well-defined expanded or contracted lattice constant, corresponding to FM or AF order, respectively) on cooling into the contracted (AF) state than on heating into the expanded (FM) state. This scenario is reminiscent of the freezing and melting at a solid $\Longleftrightarrow$ liquid phase transition.

The nominally equiatomic sample that we studied was prepared by conventional dc magnetron sputter codeposition on polished (001) periclase $(\mathrm{MgO})$ using the method described in detail in Ref. 37, although in this case the sample was uncapped. The layer thickness of $55 \mathrm{~nm}$ was confirmed by low angle X-ray reflectometry performed on a laboratory-based diffractometer. The same instrument was used to measure the $\theta-2 \theta$ diffraction pattern along the film normal, with the data shown in Fig. 1. The film can be seen to have grown epitaxially on the single crystal substrate. The presence of a (001) reflection, a forbidden reflection for the bcc structure of disordered FeRh, indicates the presence of B2 ordering. Following the procedure described by Warren, ${ }^{38}$ we determine the chemical order parameter to be $S=0.83$ for this sample. This quantity $S$ is a measure of the 


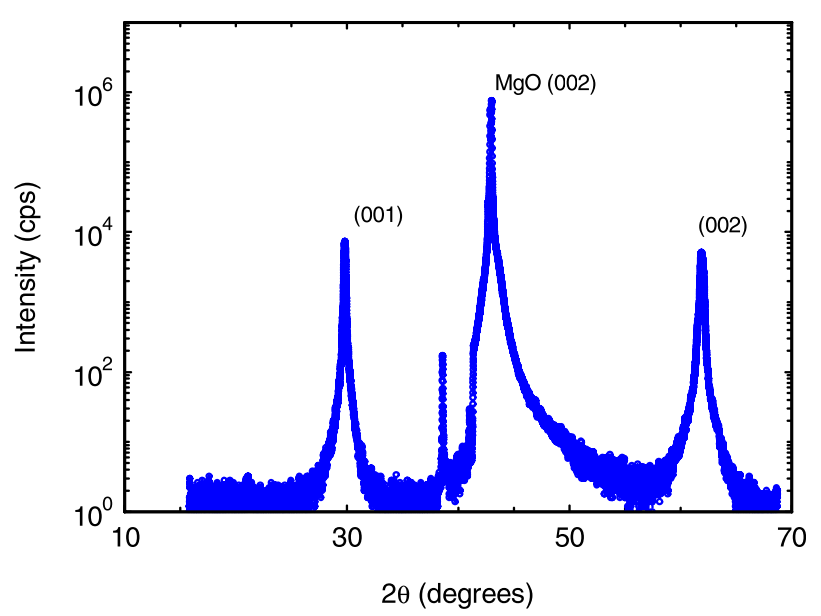

FIG. 1. Laboratory X-ray $\theta-2 \theta$ diffraction spectrum of a $55 \mathrm{~nm}$ thick FeRh layer grown on (001) $\mathrm{MgO}$. The presence of the (001) reflection, usually forbidden, demonstrates the presence of B2 ordering, with a chemical order parameter $S=0.83$.

density of anti-site defects, where pairs of $\mathrm{Fe}$ and $\mathrm{Rh}$ atoms are interchanged from their proper sublattices in the material.

Superconducting quantum interference device (SQUID) magnetometry measurements, performed at a field of $5 \mathrm{~T}$, are presented in Fig. 2, which confirmed the presence of an $\mathrm{AF} \Longleftrightarrow \mathrm{FM}$ phase transition in the FeRh epilayers. The application of this in-plane field suppressed the transition to a temperature that is within the range that our magnetometer can access. The transition is suppressed by $\sim 8 \mathrm{~K} / \mathrm{T},{ }^{8}$ implying that the zero field transition temperature is $\sim 40 \mathrm{~K}$ higher than that seen in this figure. The magnetization changed between values of about $980 \mathrm{emu} / \mathrm{cm}^{3}$ in the FM state and $10 \mathrm{emu} / \mathrm{cm}^{3}$ in the AF state. We attribute the small remanant magnetization in the nominally AF state to retained surface ferromagnetism, as previously seen both by us ${ }^{14,15}$ and Baldasseroni et al. ${ }^{17,34}$ The resistivity of the sample also changes by a factor of roughly two as the sample undergoes its magnetostructural transition, as is usual. ${ }^{11}$

High-resolution X-ray diffraction experiments were carried out at the National Synchrotron Light Source (NSLS) on beamline X22C. An X-ray photon energy of $10.0097 \mathrm{keV}$ (corresponding to a wavelength $\lambda=1.238 \AA$ ) was used. The diffractometer was equipped with a vacuum oven consisting

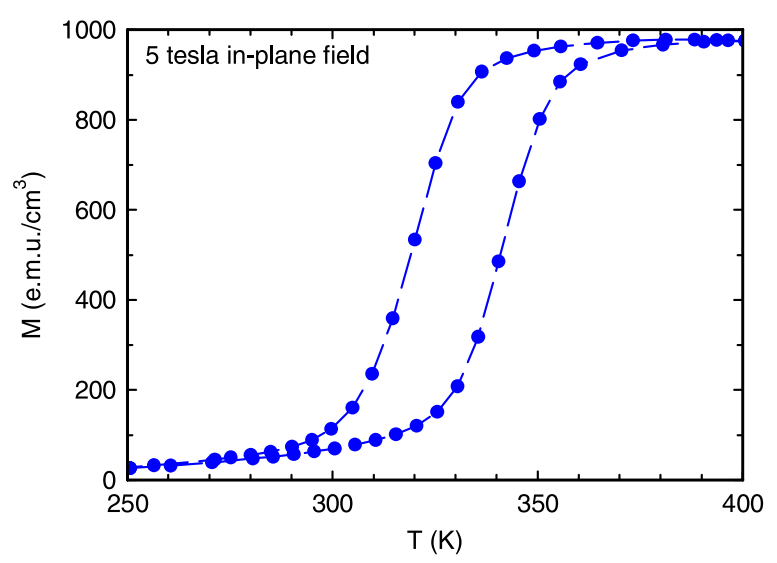

FIG. 2. Magnetization measurements as a function of temperature, measured under the application of a $5 \mathrm{~T}$ in-plane field. of a vacuum enclosure, large area boron-nitride heater, and a beryllium dome, which permitted measurement of all relevant Bragg reflections. The oven is equipped with a water jacket for stable temperature control. The measurements consisted of repeated scans through the (004) Bragg reflection of the FeRh epilayer as the temperature was varied to sweep the epilayer back and forth through its magnetostructural transition. The heating and cooling rates were of the order of $2 \mathrm{~K} / \mathrm{h}$, low enough that the experiment was effectively quasistatic. Thus, whilst we were not able to study the ultrafast dynamics of non-equilibrium phase coexistence, as is possible using femtosecond laser heating, ${ }^{22,33}$ we were able to investigate both the heating and cooling processes of the epilayer and compare them.

Data acquired during cooling into the AF, latticecontracted phase are shown in Fig. 3. The data are presented having converted the scattering angle $\theta$ into the out-of-plane lattice constant $c$, which is given by $c=\lambda l /(2 \sin \theta)$ for a reflection with Miller indices $(00 l)$. The lattice constant shrinks from a value of $3.017 \AA$ to $2.997 \AA$ as the sample passes through the transition. We can associate material with the larger lattice constant as being FM and material with the smaller lattice constant as being in the AF phase. It is to be expected that the lattice expansion is only significant in this out-of-plane direction, with the FeRh lattice constant clamped to that of the substrate in the film plane. ${ }^{31}$

Two distinct Bragg peaks can be seen in the middle of the transition (thick black curve), implying a pronounced phase separation at this temperature: the state of the sample is highly inhomogeneous, being broken up into well-defined coexisting domains where the out-of-plane lattice constant has either become largely contracted or remains largely expanded. The transition process consists of the growth of the latticecontracted domains at the expense of the lattice-expanded ones, presumably by domain wall motion. Snapshots of such a process have been observed by PEEM of the magnetism in FeRh layers. ${ }^{34-36}$ The peak widths arise from a mix of Scherrer-type broadening and the finite angular resolution of the instrument. The former indicates the vertical spatial extent

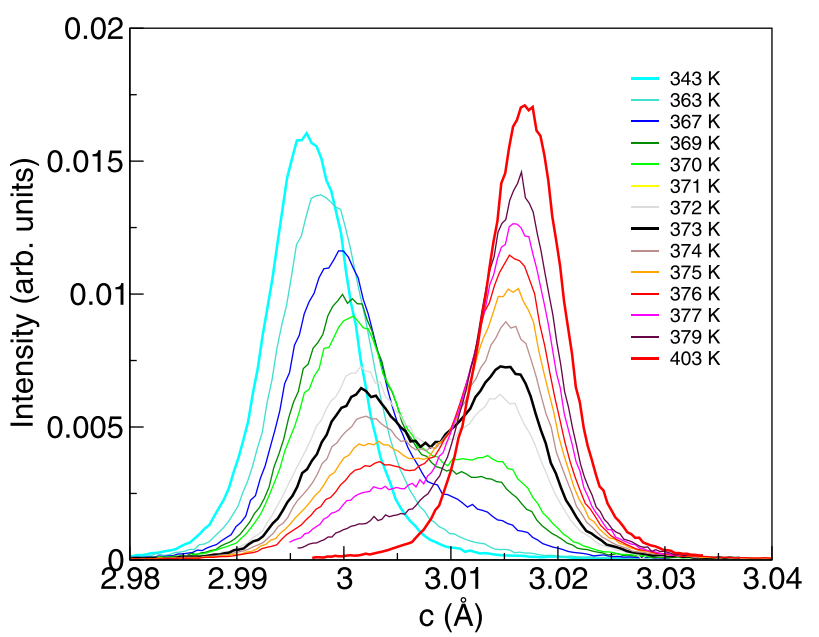

FIG. 3. Variation in out-of-plane lattice constant $c$ on cooling from $403 \mathrm{~K}$ to $343 \mathrm{~K}$, determined from the motion of the (004) reflection of a $55 \mathrm{~nm}$ thick FeRh layer grown on (001) MgO. The curve marked in black (373 K) is deemed to be the mid-point of the magnetostructural transition during cooling. 
$\xi$ of the diffracting region, given by $\xi=K \lambda / \Delta \theta \cos \theta$, where $K \approx 1.19$ for the thin film geometry. ${ }^{39}$ The latter was determined to be $0.17^{\circ}$ and can be deconvoluted from the measured peak width by subtraction in quadrature to yield the appropriate value of $\cos \theta$ for use in the Scherrer formula. Even in the middle of the transition, the deconvoluted widths imply that $\xi \gtrsim 50 \mathrm{~nm}$, roughly equal to the film thickness. This shows that these domains extend throughout the height of the film and are thus side-by-side regions of which the PEEM experiments provide a plan view. Since the scattering vector here is out of the layer plane, we have no information here about the lateral size of these regions from these measurements. (The various PEEM studies all show that they are typically $\sim 1 \mu \mathrm{m}$ across.)

In Fig. 4, we show an equivalent data set acquired during heating, where the lattice constant returns to its original position at the highest temperatures measured. We can see from these data that the transition on heating is much more homogeneous, with the separate phases more poorly defined: for instance, at the mid-point of the transition (thick black curve) the depression between the two peaks is far weaker. It is clear that the return to the FM phase on heating is not by a reversal of the same mechanism as the cooling transition to the AF phase, rigidly displaced to a higher temperature, but follows a different microscopic pathway of nucleation and growth that has a greatly reduced degree of separate phase coexistence. The quasi-expanded/contracted regions have more similar lattice constants at the heating transition midpoint than at the cooling transition mid-point, suggesting that these regions are better coupled in such as way as to strain each other more closely to the average lattice constant of the whole film.

Each curve in Figs. 3 and 4 has been fitted by a pair of Gaussian functions. This procedure allowed us to determine the peak centroid $(c)$, intensity $(I)$, and peak half-width $(\Delta c)$ as a function of temperature for each of the two phases as the heating and cooling transitions take place. The results of doing so are shown in Fig. 5, with a clear hysteresis in the transition in each parameter. Low intensity peaks, defined as

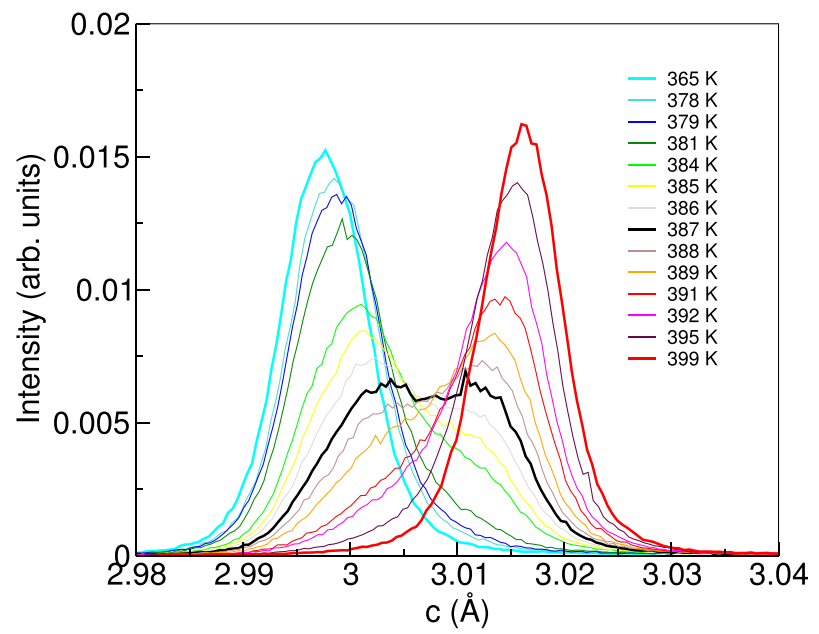

FIG. 4. Variation in out-of-plane lattice constant $c$ on heating from $365 \mathrm{~K}$ to $399 \mathrm{~K}$, determined from the motion of the (004) reflection of a $55 \mathrm{~nm}$ thick $\mathrm{FeRh}$ layer grown on (001) $\mathrm{MgO}$. The curve marked in black (387 K) is deemed to be the mid-point of the magnetostructural transition during heating.
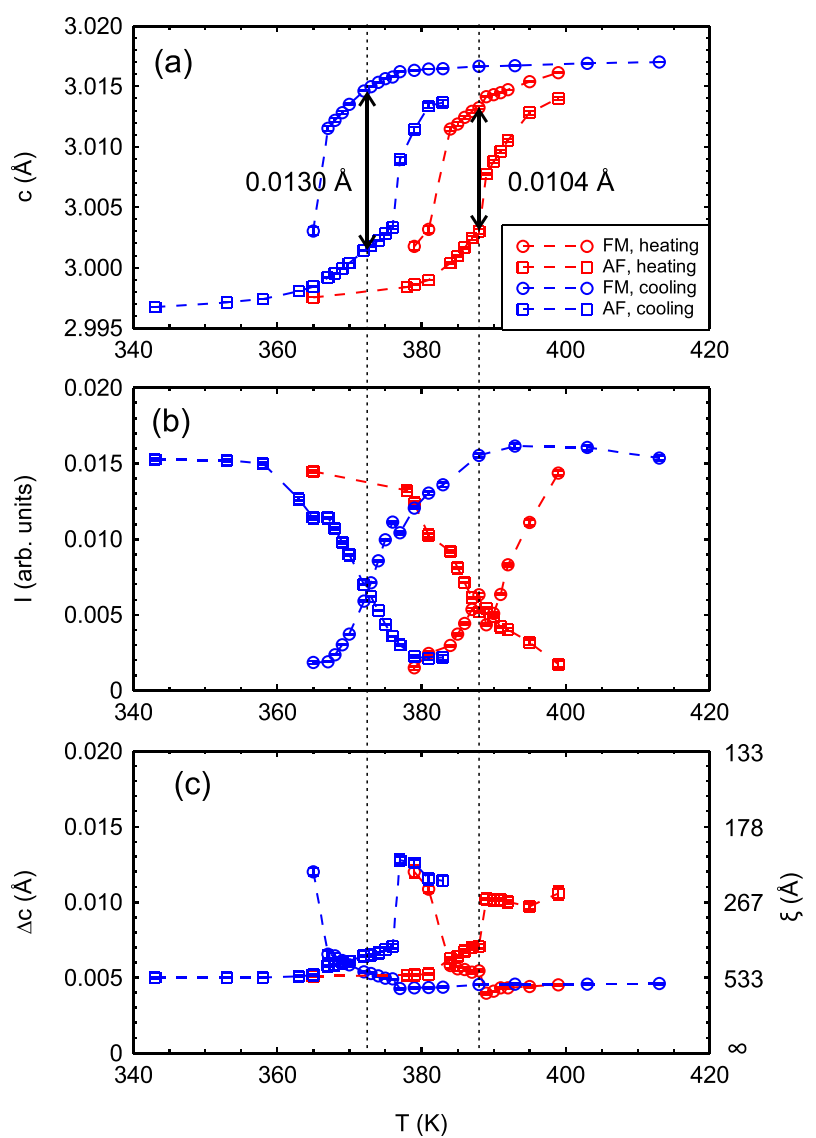

FIG. 5. Results of fitting the data in Figs. 3 and 4. The plots show (a) the lattice constant $c$, (b) the peak intensity $I$, and (c) the deconvoluted peak halfwidth $\Delta c$ (with the Scherrer coherence length $\xi$ on the right-hand ordinate axis) for the AF and FM phases for the $55 \mathrm{~nm}$ thick FeRh layer grown on (001) $\mathrm{MgO}$. The midpoints of the transitions on heating and cooling are identified as the points where the peak intensities for the two phases are equal, marked with vertical dotted lines. The vertical double-headed arrows in panel (a) show the difference in lattice constant $c$ at that point.

having an intensity smaller than the full value deep within the relevant phase by at least one order of magnitude, were neglected. The peak half-widths are shown after deconvolution of the instrumental broadening, and so are slightly smaller than in the raw data shown in Figs. 3 and 4. We define the mid-point of each transition as the temperature at which the intensities of the peaks representing the two phases are equal. These temperatures are marked in Fig. 5 with vertical dotted lines, and the data sets measured closest to these temperatures are marked with black lines in Figs. 3 and 4 . There is a small discrepancy between the transition temperatures observed here and those that can be inferred from the SQUID data in Fig. 2 with $\mathrm{a}+40 \mathrm{~K}$ offset to account for the $5 \mathrm{~T}$ field. This is due either to this assumed offset value being inexact, with the assumed correction slope not being exactly $8 \mathrm{~K} / \mathrm{T}$ for this sample, or to a small miscalibration of the thermometer in the X-ray oven. This discrepancy does not affect the analyses and conclusions of this paper.

It is then interesting to compare the different states of the sample at these equivalent points on the heating and cooling branches. The double-headed arrows in Fig. 5(a) indicate the differences in lattice constant for the two phases determined from the Bragg peak angles at these mid-points. 
The lattice constants of the two phases differ by $0.0130 \AA$ on cooling but only $0.0104 \AA$ on heating. (The uncertainty in these values is $\sim 0.0004 \AA$.) This marked difference reinforces the conclusion that there is a greater degree of phase separation-that is, well-defined regions of the sample with different lattice constants-during the cooling process from the FM state. Evidently, these regions are able to relax to close to their equilibrium lattice constants during cooling, presumably with steep strain gradients at the phase boundaries. Meanwhile, at the mid-point of the transition during heating, the lattice constant is more homogeneous throughout the material, as might be expected for more intimately mixed phases that strain each other. The Bragg peaks are also less intense at the heating mid-point than at the cooling midpoint (see Fig. 5(b)).

The peak half-widths (Figure 5(c)) are small at the midpoints, corresponding to values of $\xi$ that are comparable to the film thickness, indicating that the regions of the different phases span the height of the film in this region. In most cases, they remain so for a few kelvins on either side of each of the mid-points. When $T$ is more than $\sim 5 \mathrm{~K}$ from the midpoint, whilst the equilibrium phase still spans the whole film, the metastable phase regions yield broader peaks, and so are of restricted height $\xi$, probably in regions close to the film surfaces. The exception to this general behavior is for the AF phase during heating, where the peak broadens immediately after the transition midpoint, indicating an abrupt collapse in the vertical extent $\xi$ of the AF regions as soon as the midpoint is passed.

A canonical example of a first-order phase transition is the melting and freezing processes at a solid $\Longleftrightarrow$ liquid transition. Like all first-order phase transitions, equilibrium occurs at a temperature $T=T_{\mathrm{c}}$ when the Gibbs free energy $G=U-T S$ of a low internal energy $U$, low entropy $S$ phase (in that case, the solid) is equal to that of a high $U$, high $S$ phase (the liquid). ${ }^{40}$ (In this discussion, we neglect the magnetic term in $G$ since we are considering the situation in our FeRh layers at zero magnetic field, and also, for simplicity, neglected the change in sample volume $V$ caused by the lattice expansion.) In a first-order phase transition, the transformation from the high- $G$ phase to the low- $G$ phase when $T$ varies from $T_{\mathrm{c}}$ is inhibited by kinetic barriers that are overcome after superheating and supercooling, giving rise to a thermal hysteresis. Superheating at a solid $\Rightarrow$ liquid transition is rare, since nuclei of liquid material are generally already present at the surface of solids as the melting point is approached, meaning that there is little coexistence of separate phases during the transition proper: one phase abruptly transforms into the other as these nuclei grow rapidly. ${ }^{41} \mathrm{On}$ the other hand, when a liquid $\Rightarrow$ solid transition occurs, there is usually considerable undercooling, since nuclei of the solid phase within the supercooled liquid must exceed a critical size before they are kinetically stable. This scenario leads to spatially separated regions of the two different phases that coexist within the system.

The different warming and cooling processes we have observed in the FeRh epilayer studied here mirror this prototypical description of the freezing and melting processes in many ways. Both the degree of phase separation in the film and of undercooling or superheating differ during the cooling and heating processes. We have already described how there is a much more clearly defined separation of coexisting phases on the cooling than on the heating branch. It has also already been shown that the magnetostructural phase transformation of an FeRh epilayer proceeds from the surface into the film, ${ }^{22,31}$ in analogy with many melting processes. Moreover, our measurements here demonstrate that the heating process lacks superheating. Whilst the FM regions with a height $\xi$ that spans the film thickness persist in an undercooled state for $\sim 6 \mathrm{~K}$ below the cooling mid-point, the $\mathrm{AF}$ regions abruptly collapse to reduced- $\xi$ (probably near-surface) regions right at the mid-point: these full-height $\mathrm{AF}$ regions cannot be superheated past that temperature. On the other hand, fullheight FM domains can be undercooled below the mid-point by almost $10 \mathrm{~K}$.

To summarise, we have demonstrated that the kinetics of the magnetostructural phase transition in an FeRh epilayer differ in the heating and cooling branches, in a manner consistent with the features of canonical melting/freezing first order phase transition. Nevertheless, these results naturally raise the question of what the nature of the analogous phases must be such that melting/freezing-like kinetics can be observed. In Ref. 11, we suggested that in the AF phase a Mott-like transition has occurred for Fe $3 d$ electrons that are itinerant in the FM phase, in the presence of other itinerant electron bands that remain unaffected by the transition. We would like to note that the analogy between the kinetics of the AF-FM transition and conventional freezing-melting transitions observed here consistent with this picture if considered in terms of localization (freezing) and delocalization (melting) of that group of $\mathrm{Fe} 3 \mathrm{~d}$ (carrier) electrons. We hope our results stimulate further theoretical and experimental studies of the nature of the phase transition in this fascinating material.

We gratefully acknowledge the help and advice from $\mathrm{M}$. Ali, J. S. Weaver, and N. A. Porter in Leeds. This work was supported by a linked grant through the Materials World Network scheme by the National Science Foundation under Grant No. DMR-0908767 and the UK Engineering and Physical Sciences Research Council, Grant No. EP/ G065640/1. Use of the National Synchrotron Light Source, Brookhaven National Laboratory, was supported by the U.S. Department of Energy, Office of Science, Office of Basic Energy Sciences, under Contract No. DE-AC02-98CH10886. We would like to thank Darì Arena for assistance with the experiments at X22C and Jean Jordan-Sweet for the loan of a furnace used there.

${ }^{1}$ J. S. Kouvel and C. C. Hartelius, J. Appl. Phys. 33, 1343 (1962).

${ }^{2}$ F. de Bergevin and L. Muldawer, C. R. Acad. Sci. 252, 1347 (1961).

${ }^{3}$ A. I. Zakharov, A. Kadomtseva, R. Levitin, and E. Ponyatovskii, Zh. Eksp. Teor. Fiz. 46, 2003 (1964) [Sov. Phys. JETP 19, 1348 (1964)].

${ }^{4}$ M. P. Annaorazov, S. A. Nikitin, A. L. Tyurin, K. A. Asatryan, and A. K. Dovletov, J. Appl. Phys. 79, 1689 (1996).

${ }^{5}$ J. M. Lommel and J. S. Kouvel, J. Appl. Phys. 38, 1263 (1967).

${ }^{6}$ Y. Ohtani and I. Hatakeyama, J. Appl. Phys. 74, 3328 (1993).

${ }^{7}$ J. van Driel, R. Coehoorn, G. J. Strijkers, E. Bruck, and F. R. de Boer, J. Appl. Phys. 85, 1026 (1999).

${ }^{8}$ S. Maat, J.-U. Thiele, and E. E. Fullerton, Phys. Rev. B 72, 214432 (2005). 
${ }^{9}$ C. Stamm, J.-U. Thiele, T. Kachel, I. Radu, P. Ramm, M. Kosuth, J. Minár, H. Ebert, H. A. Dürr, W. Eberhardt, and C. H. Back, Phys. Rev. B 77, 184401 (2008).

${ }^{10}$ M. Sharma, H. M. Aarbogh, J. U. Thiele, S. Maat, E. E. Fullerton, and C. Leighton, J. Appl. Phys. 109, 083913 (2011).

${ }^{11}$ M. A. de Vries, M. Loving, A. P. Mihai, L. H. Lewis, D. Heiman, and C. H. Marrows, New J. Phys. 15, 013008 (2013).

${ }^{12}$ J.-U. Thiele, S. Maat, and E. E. Fullerton, Appl. Phys. Lett. 82, 2859 (2003).

${ }^{13}$ X. Marti, I. Fina, C. Frontera, J. Liu, P. Wadley, Q. He, R. J. Paull, J. D. Clarkson, J. Kudrnovský, I. Turek, J. Kuneš, D. Yi, J.-H. Chu, C. T. Nelson, L. You, E. Arenholz, S. Salahuddin, J. Fontcuberta, T. Jungwirth, and R. Ramesh, Nat. Mater. 13, 367 (2014).

${ }^{14}$ Y. Ding, D. A. Arena, J. Dvorak, M. Ali, C. J. Kinane, C. H. Marrows, B. J. Hickey, and L. H. Lewis, J. Appl. Phys. 103, 07B515 (2008).

${ }^{15}$ R. Fan, C. J. Kinane, T. R. Charlton, R. Dorner, M. Ali, M. A. de Vries, R. Brydson, C. H. Marrows, B. J. Hickey, D. A. Arena, B. K. Tanner, G. Nisbet, and S. Langridge, Phys. Rev. B 82, 184418 (2010).

${ }^{16}$ M. Loving, M. A. de Vries, F. Jimenez-Villacorta, C. Le Graët, X. Liu, R. Fan, S. Langridge, D. Heiman, C. H. Marrows, and L. H. Lewis, J. Appl. Phys. 112, 043512 (2012).

${ }^{17}$ C. Baldasseroni, G. K. Pálsson, C. Bordel, S. Valencia, A. A. Unal, F. Kronast, S. Nemsak, C. S. Fadley, J. A. Borchers, B. B. Maranville, and F. Hellman, J. Appl. Phys. 115, 043919 (2014).

${ }^{18}$ I. Suzuki, M. Itoh, and T. Taniyama, Appl. Phys. Lett. 104, 022401 (2014).

${ }^{19}$ R. O. Cherifi, V. Ivanovskaya, L. C. Phillips, A. Zobelli, I. C. Infante, E. Jacquet, V. Garcia, S. Fusil, P. R. Briddon, N. Guiblin, A. Mougin, A. A. Ünal, F. Kronast, S. Valencia, B. Dkhil, A. Barthélémy, and M. Bibes, Nat. Mater. 13, 345 (2014).

${ }^{20}$ G. Ju, J. Hohlfeld, B. Bergman, R. J. M. van de Veerdonk, O. N. Mryasov, J.-Y. Kim, X. Wu, D. Weller, and B. Koopmans, Phys. Rev. Lett. 93, 197403 (2004).

${ }^{21}$ F. Quirin, M. Vattilana, U. Shymanovich, A. El-Kamhawy, A. Tarasevitch, J. Hohlfeld, D. von der Linde, and K. Sokolowski-Tinten, Phys. Rev. B 85, 020103 (2012).

${ }^{22}$ S. O. Mariager, F. Pressacco, G. Ingold, A. Caviezel, E. Möhr-Vorobeva, P. Beaud, S. L. Johnson, C. J. Milne, E. Mancini, S. Moyerman, E. E. Fullerton, R. Feidenhans'1, C. H. Back, and C. Quitmann, Phys. Rev. Lett. 108, 087201 (2012).
${ }^{23}$ C. Koenig, J. Phys. F: Met. Phys. 12, 1123 (1982).

${ }^{24}$ V. L. Moruzzi and P. M. Marcus, Phys. Rev. B 46, 2864 (1992).

${ }^{25}$ R. Y. Gu and V. P. Antropov, Phys. Rev. B 72, 012403 (2005).

${ }^{26}$ L. M. Sandratskii and P. Mavropoulos, Phys. Rev. B 83, 174408 (2011).

${ }^{27}$ J. B. Staunton, R. Banerjee, M. dos Santos Dias, A. Deak, and L. Szunyogh, Phys. Rev. B 89, 054427 (2014).

${ }^{28}$ D. W. Cooke, F. Hellman, C. Baldasseroni, C. Bordel, S. Moyerman, and E. E. Fullerton, Phys. Rev. Lett. 109, 255901 (2012).

${ }^{29}$ A. X. Gray, D. W. Cooke, P. Krüger, C. Bordel, A. M. Kaiser, S. Moyerman, E. E. Fullerton, S. Ueda, Y. Yamashita, A. Gloskovskii, C. M. Schneider, W. Drube, K. Kobayashi, F. Hellman, and C. S. Fadley, Phys. Rev. Lett. 108, 257208 (2012).

${ }^{30}$ R. Barua, F. Jiménez-Villacorta, and L. H. Lewis, Appl. Phys. Lett. 103, 102407 (2013).

${ }^{31}$ J. W. Kim, P. J. Ryan, Y. Ding, L. H. Lewis, M. Ali, C. J. Kinane, B. J. Hickey, C. H. Marrows, and D. A. Arena, Appl. Phys. Lett. 95, 222515 (2009).

${ }^{32}$ M. Loving, F. Jimenez-Villacorta, B. Kaeswurm, D. A. Arena, C. H. Marrows, and L. H. Lewis, J. Phys. D: Appl. Phys. 46, 162002 (2013).

${ }^{33}$ I. Radu, C. Stamm, N. Pontius, T. Kachel, P. Ramm, J.-U. Thiele, H. A. Dürr, and C. H. Back, Phys. Rev. B 81, 104415 (2010).

${ }^{34}$ C. Baldasseroni, C. Bordel, A. X. Gray, A. M. Kaiser, F. Kronast, J. Herrero-Albillos, C. M. Schneider, C. S. Fadley, and F. Hellman, Appl. Phys. Lett. 100, 262401 (2012).

${ }^{35}$ S. O. Mariager, L. Le Guyader, M. Buzzi, G. Ingold, and C. Quitmann, e-print arXiv:1301.4164v1 [cond-mat.mtrl-sci].

${ }^{36}$ C. Kinane, M. Loving, M. A. de Vries, R. Fan, T. Charlton, J. Claydon, D. A. Arena, F. Maccherozzi, S. Dhesi, D. Heiman, C. H. Marrows, L. H. Lewis, and S. Langridge, "Thermal hysteresis asymmetry in antiferromagnetic/ferromagnetic phase coexistence in Pd doped binary alloy FeRh thin films," (unpublished).

${ }^{37}$ C. Le Graët, M. A. de Vries, M. McLaren, R. M. D. Brydson, M. Loving, D. Heiman, L. H. Lewis, and C. H. Marrows, J. Vis. Exp. 80, e50603 (2013).

${ }^{38}$ B. E. Warren, X-Ray Diffraction (Addison-Wesley, Reading, Mass, 1969).

${ }^{39}$ J. I. Langford and A. J. C. Wilson, J. Appl. Crystallogr. 11, 102 (1978).

${ }^{40} \mathrm{P}$. Papon, J. Leblond, and P. H. E. Meijer, The Physics of Phase Transitions, 2nd ed. (Springer, Berlin, 2006).

${ }^{41}$ M. de Podesta, Understanding the Properties of Matter, 2nd ed. (Taylor and Francis, London, 2002). 